\title{
A Bound for an Interval of the Dirichlet Series for the Zeta-Function of a Quadratic Form on the Unit Line
}

\author{
I. F. Avdeev \\ Presented by Academician V.A. Il'in February 12, 2014
}

Received February 12, 2014

DOI: $10.1134 / \mathrm{S} 106456241405007 \mathrm{X}$

The main result of this paper is the application of Vinogradov's method to estimate the zeta-function $\zeta(s, K)$ of a quadratic form $K$ with increasing negative discriminant $(-d)$ [1]. We effectively apply this method by using Voronin's approach to derive approximations by intervals of the series for the zeta-function of a quadratic form [2]. The principal term of this approximation is the initial interval of the Dirichlet series of the zeta-function under consideration, whose terms are not "twisted" with any character. This makes it possible to reduce the problem to estimating the double zeta sum.

We state the following theorem on a bound for the zeta-function of a quadratic form on the unit line.

Theorem. Let $K(m, n)$ be the quadratic form

$$
K(m, n)=4 a\left(a m^{2}+b m n+c n^{2}\right)
$$

with integer coefficients $a, b$, and $c$ satisfying the conditions

$$
\begin{gathered}
|b| \leq a \leq c, \\
d=4 a c-b^{2}>0 .
\end{gathered}
$$

Let $f(m, n)=(K(m, n))^{-s}$, where $s=1+i t, t>0, t \rightarrow \infty$. Then the sums $S$ of the form

are bounded as

$$
S=\sum_{m, n \ll t} f(m, n)
$$

$$
S \ll \frac{(\ln t)^{2 / 3}}{a \sqrt{d}} .
$$

The proof of this theorem is based on estimating double zeta sums. Our scheme of reasoning is close to that in [3, p. 332]. An important role in the proof is played by the following lemma of independent interest.

Lemma. Let $V(t, M, N)$ be a sum of the form

$$
=\sum_{N \leq n \leq 2 N} \sum_{M \leq n \leq 2 M}\left((2 a m+b n)^{2}+d n^{2}\right)^{-i t}
$$

where $M, N \ll t$. Then, for some rsatisfying the condition $0 \leq \gamma<\frac{1}{3}$, the sum $V(t, M, N)$ satisfies the inequality

$$
|V(t, M, N)| \ll M N \exp \left(-\gamma \frac{\ln ^{3}(N+M)}{\ln ^{2} t}\right) .
$$

\section{REFERENCES}

1. I. M. Vinogradov, Selected Works (Akad. Nauk SSSR, Moscow, 1952) [in Russian].

2. S. M. Voronin, Selected Works: Mathematics (Mosk. Gos. Tekhn. Univ. im. N.E. Baumana, Moscow, 2006) [in Russian].

3. G. I. Arkhipov, Selected Works (Orlovsk. Gos. Univ., Orel, 2013) [in Russian].

Translated by $O$. Sipacheva

Orel State University, Komsomol'skaya ul. 95,

Orel, 302026 Russia

e-mail:ivan_avd@mail.ru 